\title{
Esophageal stenting using a newly marketed dedicated anchoring device
}

\section{Vincent Zimmer ${ }^{\mathrm{a}, \mathrm{b}}$}

Marienhausklinik St. Josef Kohlhof, Neunkirchen; Saarland University Medical Center, Saarland University, Homburg, Germany

Esophageal self-expanding metal stent (SEMS) insertion as the mainstay palliative treatment for advanced malignancy is safe with high reported technical success rates. However, its long-term technical and clinical efficacy might be questioned, given high rates of migration, e.g. when using fully and/or partially covered SEMS designs as first-line options. Against this background, techniques of stent anchoring have evolved in recent years; however, dedicated endoscopic accessory technology has not been available so far. The recently marketed and FDA-approved stentfixOTSC as an innovative iteration of the over-the-scopeclip (OTSC) platform (stentfix OTSC, Ovesco Endoscopy AG, Tübingen, Germany) closes this technological gap [1]. Because of its ease of application, the use of the OTSC has become widely disseminated, with most interventional endoscopists being well trained and acquainted with the OTSC technology. Thus, this specifically designed anchoring device, which has a distinct clip design with indentations in the application cap and clip brackets bowed to the outside to better engage stent ends, may become a novel endoscopic standard-of-care, although rigorous systematic study of its technical and clinical efficacy in the short and long term is clearly needed.

The use of the anchoring device is illustrated in this case of a 91-year-old male who presented with dysphagia due to

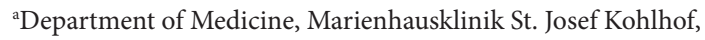
Neunkirchen (Vincent Zimmer); ${ }^{b}$ Department of Medicine II, Saarland University Medical Center, Saarland University, Homburg (Vincent Zimmer), Germany

Conflict of Interest: None

Correspondence to: Dr. Vincent Zimmer, Department of Medicine, Marienhausklinik St. Josef Kohlhof, Klinikweg 1-5, 66539 Neunkirchen, Germany, e-mail: vincent.zimmer@gmx.de

Received 13 November 2020; accepted 24 November 2020 published online 27 January 2021

DOI: https://doi.org/10.20524/aog.2021.0587
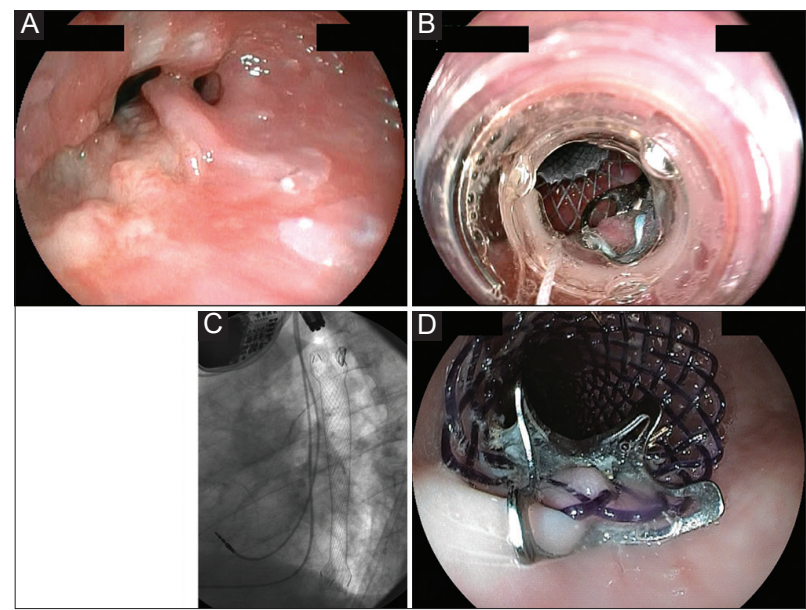

Figure 1 (A) Malignant distal esophageal stricture due to adenocarcinoma prior to stent implantation using a pcSEMS. (B) Endoscopic and (C) fluoroscopic demonstration of technical success in stentfixOTSC application to proximal stent struts. (D) Juxtaposed illustration of a conventional OTSC system fixed to the proximal parts of a biodegradable stent for refractory benign esophageal stricture (note differentially bowed clip brackets compared to B)

SEMS, self-expanding metal stent; OTSC, over-the-scope clip

distal esophageal adenocarcinoma (Fig. 1A). An uncomplicated stent deployment was performed using a $100 \times 24 \mathrm{~mm}$ pcSEMS with ancillary stentfixOTSC anchoring of the proximal stent struts (Fig. 1 B,C). For comparison purposes, Fig. 1D shows a biodegradable stent being fixed by a conventional OTSC system.

\section{Reference}

1. Perumpail RB, Muthusamy VR. Gastrointestinal stents: thinking outside the box. Tech Innov Gastrointest Endosc 2020;22:239-244. 